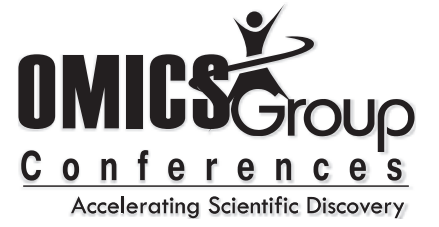

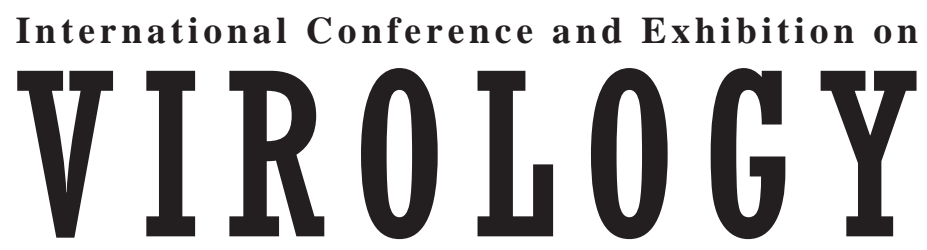

5-7 September 2011 Baltimore, USA

\section{Systemic alteration of Cholesterol Homeostasis in experimental and natural scrapie}

Alessandra Pani', $\mathbf{S}$. Vascellari', C.D. Orru', C. Abete', A. Mandas ${ }^{2}$, S. Dessi ${ }^{2}$ 'Department of Biomedical Science \& Technology

${ }^{2}$ Department of Internal Medicine, University of Cagliari, Italy ver the last few years a significant amount of genetic, biochemical, and pharmacological data have indicated that prion disorders are associated with brain cholesterol alterations. At the molecular level, growing evidence indicate that the cholesterol-rich domains of plasma membrane (i.e. rafts) represent preferred sites of conversion of the cellular prion protein (PrPc) into pathogenic prion isoform(s) (PrPsc), and that alterations of membrane cholesterol, the main regulator of organization and function of raft domains, play a key role in prion pathophysiology. In this context, our studies showed that brains from mice and sheep infected with scrapie, as well as persistently prion-infected mouse neuroblastoma N2a cells, exhibit an increased content of cholesterol and an altered ratio of the intracellular cholesterol pools [i.e. free cholesterol (FC) vs. cholesterol esters (CE)], resulting in abnormal cytoplasmic accumulation of CE. Interestingly, we found that also cells from peripheral tissues [i.e. ex vivo cultures of skin fibroblasts and peripheral blood mononuclear cells (PBMCs)] of scrapie-infected sheep, show a similar pattern of cholesterol alterations. The biochemical cholesterol modifications were accompanied by changes in the expression levels of genes and gene-products involved in cholesterol esterification and trafficking [i.e. acyl-coenzymeA:cholesterol-acyltransferase (ACAT-1; up-regulated) and caveolin-1 (Cav-1; down-regulated)]. Altogether these data indicate that prion disorders are likely associated with major systemic alterations of the cholesterol homeostatic networks. In addition, in persistently prion-infected N2a cells, we found that drugs [i.e. everolimus, pioglitazone, Sandoz 58035, progesterone, verapamil] able to reduce the CE pool by different mechanisms, were also able to inhibit the generation of the PK-resistant prion protein (PrPres) in a dose-response manner, and that strong synergistic anti-prion effects can be obtained with combinations of various cholesterol interfering drugs, apparently via restoration of cholesterol homeostasis in the infected cell. Although effectiveness of such drug combinations has yet to be proved in vivo, the enhanced anti-prion effect suggests that a rational design of drug combinations which interact at the cholesterol metabolism level, contributing to a better understanding of the complex relationship that links cholesterol metabolism to PrPsc biogenesis, may lead to the development of novel therapeutic and/or prophylactic approaches for the successful control of prion disorders.

\section{Biography}

Alessandra Pani has completed her PhD at the age of 25 years from the University of Cagliari and postdoctora studies from the Department of Virology, London Hospital Medical College in London, and the Department of Molecular and Cell Biology, University of Connecticut, in Storrs, USA, School of Medicine. She is the coordinator of the PhD Course in R\&D of Antiviral Drugs of the University of Cagliari. She has published more than 60 papers in reputed international journals and is co-inventor of 9 patents and patent applications. 